\title{
Nomoto Indices for Constant-Depth Zigzag Manoeuvres of an Autonomous Underwater Vehicle
}

\author{
Farhood Azarsina ${ }^{1}$ and Christopher D. Williams ${ }^{2}$ \\ ${ }^{1}$ Department of Marine Industries, Faculty of Marine Science and Technology, Science and Research Branch, Islamic Azad University, \\ Tehran 1477893855, Iran \\ ${ }^{2}$ National Research Council Canada, Institute for Ocean Technology, P.O. Box 12093, St. John's, NL, Canada A1B 3T5
}

Correspondence should be addressed to Farhood Azarsina; farhood.azarsina@mun.ca

Received 3 October 2012; Accepted 11 November 2012

Academic Editors: M. Lipinski and J. L. Zhou

Copyright (C) 2013 F. Azarsina and C. D. Williams. This is an open access article distributed under the Creative Commons Attribution License, which permits unrestricted use, distribution, and reproduction in any medium, provided the original work is properly cited.

\begin{abstract}
A two-dimensional simulation code is used to study the characteristics of constant-depth zigzag manoeuvres of the axisymmetric autonomous underwater vehicle (AUV) MUN Explorer. Sea trials data for several manoeuvres with the AUV have been reported during the past four years; however, to obtain a more complete understanding of the vehicle's hydrodynamics, additional towing tank tests and computer simulation were performed. The present work, based on the towing tank test results and sea-trials data, utilizes computer simulations to predict the performance of the MUN Explorer AUV during horizontal zigzag manoeuvres. Next, the Nomoto indices for this AUV during constant-depth zigzag manoeuvres are estimated using the simulation results, and, then, Nomoto's first-order model for the rate of turn of the vehicle during horizontal zigzag manoeuvres in response to a square-wave input for the rudder deflection angle is analytically solved. The paper investigates the validity of the simplified yaw equation to predict a zigzag manoeuvre. Results of this research are a first step to understand the details of zigzag manoeuvres of an AUV such as duration of the first execute, yaw-checking ability, and duration of the overshoot.
\end{abstract}

\section{Introduction}

The zigzag manoeuvre, also known as the Kempf overshoot or " $\mathrm{Z}$ " manoeuvre, is indicative of the ability of a marine vehicle's rudders to control the vehicle [1]. According to the recommended procedures by the ITTC [2], a "zigzag manoeuvre is obtained by reversing the rudder alternately by $\delta$ degrees to either side at a deviation $\psi$ from the initial course." The cycle of deflecting the rudders alternately can be continued through the second, the third, or more executes. The characteristics up to as far as the first overshoot are indicative of the transient response of the vehicle during a zigzag, and, during the subsequent executes, the steady-state measures of the zigzag are observed.

The quickness of response of the vehicle to the commanded rudder movement is indicated by the time $t_{a}$, the duration of the first execute also named "reach," and yawchecking ability is indicated by the time $t_{s}$, the duration of the overshoot [1, page 319].
Perrault et al. [3], using a computer model of an axisymmetric underwater vehicle, examined the sensitivity of the response of the underwater vehicle C-SCOUT during turning circles and horizontal and vertical zigzag manoeuvres to variations (or uncertainty) in hydrodynamic parameters such as added mass, the lift and drag forces on constituent components, and the point of application of the lift and drag forces.

Moreira and Soares [4] used data for a series of manoeuvres with varying rudder deflection angles and approach speeds to train and validate a recursive neural network (RNN) manoeuvring simulation model for surface ships to predict tactical circles and zigzags.

Based on the extended Nomoto equations, numerical simulations of the zigzag motion focusing on the case of directionally unstable ships were studied by Sutulo and Soares [5]. Study of the responses to the harmonic rudder motion revealed the transition from symmetric to biased yawing 
when the rudder angle amplitude drops below a certain value depending on the manoeuvring frequency.

Peréz and Clemente [6] used a nonlinear numerical model to simulate turning circle and zigzag manoeuvres for a sample ship and studied the influence of some important ship parameters at the design stage on its manoeuvrability characteristics. It was concluded that the effects of ship parameter variations on the zigzag manoeuvre are small except for variations in ship length.

Finally, related to the present study, the report by Journée [7] should be mentioned: using Nomoto's first-order model, a large number of zigzag manoeuvres were calculated at a practical range of $K$ and $T$ values (these parameters are defined later in this paper). These data were analyzed, and the relations between the zigzag manoeuvring characteristics and the Nomoto indices $K$ and $T$ were reflected in graphs.

The MUN Explorer is a survey-class AUV that was acquired by the Memorial University of Newfoundland, in June 2006. A set of manoeuvring experiments was performed using the AUV during the summer of 2006 [8] and several later trials. However, to obtain a more complete understanding of the vehicle's hydrodynamics, additional towing tank tests and computer simulation are required, because (i) performing sea trials is costly and exposes risks to the vehicle, (ii) data such as forces and moments are not measured during a free-running trial, and (iii) the effects of environmental loads and control actions on the manoeuvring are inseparable during sea trials.

In this paper, a two-dimensional simulation code is introduced and used to study the characteristics of constant-depth zigzag manoeuvres of the MUN Explorer AUV. Azarsina and Williams [9], using this planar simulation code, reported that the hydrodynamic forces that are exerted on the AUV during a turning manoeuvre vary nonlinearly as the rudder deflection angle increases and as the manoeuvre becomes more abrupt in terms of the turning rate $r$ and the radius of turn $R$.

The results reported in this paper are therefore as follows.

(a) Explorer-specific simulator validated by at-sea manoeuvres with the full-scale AUV.

(b) Generic component build-up simulator based on model-scale PMM measurements and validated by full-scale experiments with the MUN Explorer AUV.

(c) Insight into the role of Nomoto indices in prediction of the behaviour of an AUV which executes zigzag manoeuvres.

(d) Insight into the role of Nomoto indices in teaching, designing, and testing control algorithms for specific missions. For example, constant-depth trajectory with minimized response to environmental disturbances such as surface waves, constant-altitude trajectory with minimal overshoot when depth changes are commanded during surveys, and most rapid response when changing pitch attitude and depth during obstacle-avoidance manoeuvres.

\section{Characteristics of Horizontal Zigzags}

The principal numerical measures during a zigzag manoeuvre are (i) the time to reach the second execute yaw angle, $t_{a}$ (ii) the overshoot yaw angle, $\alpha_{s}$ (iii) the overshoot width of path, $d_{s}$ [1, page 206], and (iv) yaw-checking ability, $t_{s}$, which is the duration of the overshoot. The first parameter indicates the ability of the vehicle to rapidly change its course. The second and the third parameters indicate how much a helmsman is required while operating such a vessel in restricted waters. Note that if the alternating rudder angle, the yaw angle of the ship, and its distance normal to original path are all drawn on the same plot, one can compare the time lags between those variables.

The Nomoto simplified analysis of $K$ and $T$ indices, which may be obtained from zigzag trials data and are useful in comparing course keeping and turning abilities of a ship, was presented in [1, section 5.4, page 207]. Nomoto [10] divided the nondimensional simplified yaw equation of motion by the yaw damping coefficient $N_{r}^{\prime}$ and rewrote that equation as follows:

$$
T^{\prime} \dot{r}+r^{\prime}=K^{\prime} \delta .
$$

Clearly, the multiplier of yaw rate in (1) is unity, since the equation of motion was divided by the yaw damping coefficient. In dimensional form, the equation is

$$
T \dot{r}+r=K \delta,
$$

where the nondimensional parameters in (1) are related to the dimensional Nomoto parameters $T$ and $K$ in (2) as follows [1], page 207:

$$
T^{\prime}=\frac{T U}{L}, \quad K^{\prime}=\frac{K L}{U},
$$

where $U$ is the forward speed of the vehicle and $L$ is its overall length.

For a simple case where the rudder is put over suddenly to an angle $\delta_{0}$ and held there (a step-function input), the yaw rate $r$ in (2) is defined by a first-order linear differential equation which has the following solution:

$$
r=K \delta_{0}\left(1-e^{-t / T}\right) .
$$

This solution shows that the yaw rate increases exponentially, with a rate that depends on $T$ and asymptotes to a steady value $K \delta_{0}$. In [1, page 207], it is concluded that "a larger $K$ thus provides greater steady-state turning ability, and a smaller value of $T$ provides a quicker initial response to the helm." Briefly, the manoeuvring qualities of a ship are characterized using the Nomoto indices as follows:

(i) increasing $T$ improves the course stability;

(ii) decreasing $T$ improves the responsiveness to rudder;

(iii) increasing $K$ improves the turning ability.

A highly manoeuvrable ship has a large ratio of $K / T$. Also, Nomoto [11] showed that the overshoot angle during a zigzag test is, for a given rudder angle, proportional to the product KT. 


\section{The Two-Dimensional Simulation Code}

Based on experimental results for a generic axisymmetric hull shape that were measured at the Institute for Ocean Technology, National Research Council Canada (NRC-IOT), a simulation code to predict the manoeuvring characteristics of MUN Explorer AUV in the $x-y$ plane was developed via the following steps. First, the hydrodynamic axial and lateral forces and the yaw turning moment that are exerted on the bare hull of an axisymmetric underwater vehicle were modeled using the towing tank test results which show clearly the effect of hull length-to-diameter ratio. Second, the lift and drag forces that are produced by the four aft control surfaces of the AUV were modeled, which effectively accounts for the X-configuration of the stern planes. Third, the thrust force that is produced by the two-bladed single propeller of the AUV was modeled using the curve of the vehicle speed versus the propeller RPM obtained from the sea trials. Finally, manoeuvring simulations were performed, and the simulation code was tuned in order to minimize the error in turning diameter relative to the diameters measured during sea trials of the AUV. Also, the simulation results for the turning manoeuvres were compared to the theoretical formulae for turn radius and drift angle which are based on the linearized equations of motion [9]. In the following sections, a summary of this procedure is explained, and, then, the results of the simulation code to perform horizontal zigzags are presented. The simulation model provides a check on the validity of the simplified Nomoto equation to predict zigzag manoeuvres.

3.1. Bare Hull Hydrodynamics: Towing Tank Tests. Manoeuvring experiments were performed with five slender axisymmetric bare hulls in the $90 \mathrm{~m}$ long, $12 \mathrm{~m}$ wide towing tank at the NRC-IOT. The original bare hull of the underwater vehicle was about $1.6 \mathrm{~m}$ long and $0.2 \mathrm{~m}$ in maximum diameter, and the other four hulls had midbody extensions added to the original hull which made a range of length-to-diameter ratios from 8.5 to 12.5. Straight-ahead resistance runs were performed for the five bare hulls at fixed forward speeds of 1 , 2,3 , and $4 \mathrm{~m} / \mathrm{s}$. Also, static yaw runs were performed using a fixed sequence of yaw (drift) angles $\beta$ from $-2^{\circ}$ to $+20^{\circ}$ in steps of two degrees; all runs were performed at a fixed speed of $2 \mathrm{~m} / \mathrm{s}$. Analyzing the test results for the five bare hulls, implying the effect of forward speed from the straightahead test results, equations to model the drag, lift, and yaw moment coefficients were obtained based on the yaw angle and the length-to-diameter ratio of the vehicle.

3.2. Control Planes. The MUN Explorer AUV is shown in Figure 1; its overall length is about 4.5 metres, and it has a maximum diameter of about $0.7 \mathrm{~m}$. Manoeuvring of the vehicle is facilitated by four aft planes arranged in an " $\mathrm{X}$ " configuration and two foreplanes which assist with precise depth and roll control. The planes have the symmetrical cross-section of NACA 0024 airfoil and are about 35 by $35 \mathrm{~cm}$ in chord and span, that is, an aspect ratio of one.

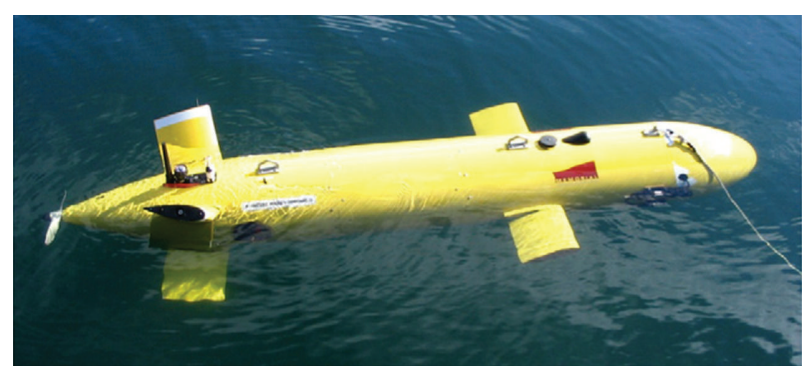

FIgURE 1: The MUN Explorer AUV being towed in water in preparation for the sea trials [12].

The numbering of the six control planes is compatible with the manufacturer's manual, in which the two bow planes are numbered 1 and 2 and the stern planes are numbered 3 and 4 on the port side and 5 and 6 on the starboard side. All planes have a positive deflection angle $\delta$ when the leading edge turns upward. Thus, the lift force of each plane is positive upward. The constant angle of stern planes' configuration is 45 degrees. The drag and lift forces produced by each plane, which depends on the incidence angle, should be projected along the $x$-axis and the $y$-axis of the vehicle coordinate system to obtain the net axial force and sway force that are produced by the control planes. More explanation about how the control planes as well as the propulsion were modeled is available in [9].

3.3. Verification of the Simulation Results. Ten runs of turning circle manoeuvres with an approach speed of $1 \mathrm{~m} / \mathrm{s}$ at a constant depth of $3 \mathrm{~m}$ that were reported by Issac et al. [8] were used to tune the response of the simulation model for the radius of turn $R$ and rate of turn $r$. The relative errors between the test and simulation results in the radius of turn and the rate of turn for those ten runs varied between $10 \%$ and $35 \%$. At the time of the sea trials, the location of the CG was not known, so the longitudinal location of the CG was approximated: $x_{G}=0.08 \mathrm{~m}$. Then, simulation results could be adjusted to about zero relative error by correcting the assumed position of the centre of gravity of the vehicle [9]. After calibration, to verify the model, simulation results for the steady radius of turn and the steady drift angle were compared to the solutions of the linearized equations of motion for a vessel during the steady phase of a turning manoeuvre which have been presented in [1, page 484].

3.4. Simulating Horizontal Zigzag Manoeuvres. To perform the horizontal zigzags, the key point is to determine when the desired heading angle is reached. As soon as the heading angle of the vehicle reaches the desired value, the rudder deflection angle should be reversed. There are three definitive stages during every zigzag simulation run.

(i) The first stage is while the vehicle is accelerating at a constant propeller RPM to reach a steady forward speed. The criterion for terminating the first stage is that the surge acceleration reduces to 0.2 percent of the maximum surge acceleration. For example, 


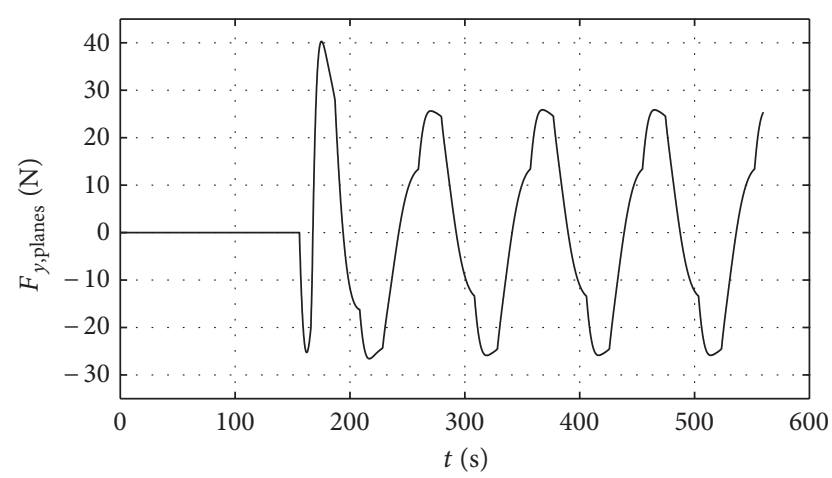

FIGURE 2: Net sway force that is produced by the four stern planes during a 10-20 zigzag simulation run.

$174 \mathrm{RPM}$ and $232 \mathrm{RPM}$ result in 1.5 and $2 \mathrm{~m} / \mathrm{s}$ steady forward speeds which, respectively, are reached after 156 and 116 seconds.

(ii) As soon as the desired approach speed is attained, the rudders (stern planes) begin to deflect at a rate of $1 \mathrm{deg} / \mathrm{s}$. If the commanded deflection angle is reached but the desired heading angle is not, then the deflection angle is kept constant at a specified value until the error in heading angle reaches a value of about one percent; the error in heading angle is defined as follows:

$$
\operatorname{error}_{\psi}=\frac{\psi-\operatorname{command}_{\psi}}{\operatorname{command}_{\psi}} .
$$

As soon as the error defined in (5) becomes less than about one percent, the rudder deflection angle is reversed at the same rate of $1 \mathrm{deg} / \mathrm{s}$.

(iii) During the zigzag, all the variables are changing in a harmonic pattern, the amplitude of which is different between the first execute (transient values) and the later executes (steady-state values). This amplitude difference is larger for larger rudder command angles. For example, during a 10-20 (10 deg rudder, $20 \mathrm{deg}$ heading) zigzag simulation run, the net sway force that is produced by the four stern planes, shown in Figure 2, has a maximum value of about $40 \mathrm{~N}$ which occurs during the first execute and (maximum) amplitude of about $26 \mathrm{~N}$ which is the amplitude observed during the third and later executes.

\section{Results of Simulations for Horizontal Zigzag Manoeuvres}

4.1. Free-Running Sea Trials. To perform a free-running zigzag manoeuvre with the MUN Explorer AUV, the rudder angle and the forward speed are the control inputs; however, the AUV control software does not allow direct control of the rudder angles. Therefore, alternately, zigzag trajectories were planned by defining the way points of the vehicle. A zigzag manoeuvre at a constant-depth of three metres at, for example, commanded amplitude and cycle-length of $20 \mathrm{~m}$ and $80 \mathrm{~m}$, respectively, was performed. It was found that there is an overshoot of approximately $8 \mathrm{~m}$ in the amplitude value, while the cycle-length was maintained at $80 \mathrm{~m}$. A total of six horizontal zigzag manoeuvres were completed during the test period [13]. It may be inferred that the AUV which acts autonomously in adjusting its control surfaces does not provide an opportunity to perform manoeuvring trials according to their conventional definition. According to Issac et al. [13], it appears that there are periods of constant heading during horizontal zigzag trials with the AUV, while, in a conventional zigzag manoeuvre, the heading angle changes continuously forming a sinusoidal pattern. "These periods of constant heading correspond to the portion of the trajectory between two waypoints where the vehicle travels for a considerable distance (16 times length overall of the AUV) in a straight line."

4.2. Verifying the Horizontal Zigzag Simulations. Four of the horizontal zigzag trials, that were performed by defining the waypoints of the MUN Explorer AUV, as reported by Issac et al. [13], are used to verify the present simulation model. Plots of the zigzag path, the AUV heading angle and the rudder deflection angle are available [13]. The maximum heading angle during a zigzag of amplitude $A_{z}$ and length $L_{z}$ may be estimated using the following formula:

$$
\psi_{0}=\frac{2 \pi A_{z}}{L_{z}} \text { in radians. }
$$

The simulation code was used to produce the same amplitude and length of zigzag at the same approach speed as in sea trials, by adjusting the rudder deflection angle. Also, the commanded heading angle is calculated using (6) for the amplitude and length of zigzags of sea trials. Results are shown in Table 1. In addition that the amplitude and length of zigzags of the trials are closely obtained, also the time period of zigzags in column 5 , Table 1 , may be compared with the sea trials. For test number $3\left(A_{z}=20\right.$ and $\left.L_{z}=160 \mathrm{~m}\right)$, at approach speed $1.5 \mathrm{~m} / \mathrm{s}$, period of the sea trial is about 120 seconds, and for test number 4, it is about 90 seconds [13]. Simulation values are 137 and 105 s, respectively. Therefore, it is verified that the simulation results are in range compared to the actual AUV. Table 1 provides information such as reach time, overshoot angle, and width of path as well as the amplitude of turning rate and sway velocity of the AUV.

Figure 3 shows the lateral position and the heading angle of the AUV during a horizontal zigzag manoeuvre at approach speed $2 \mathrm{~m} / \mathrm{s}$, rudder deflection $2.6 \mathrm{deg}$, and commanded heading $45 \mathrm{deg}$ which corresponds to test number 4 in Table 1. Also, Figure 4 shows the AUV heading and rudder deflection during the same manoeuvre. Note that the $x-y$ path of a zigzag manoeuvre does not necessarily alternate between negative and positive amplitudes, as was planned during sea trials; instead, at a slow zigzag (small rudder deflections) as shown in Figure 3, the blue curve rises to a maximum and turns back close to zero and so on. According to simulation results, for rapid zigzags (large rudder deflections), the $y$ coordinate of the vehicle, not necessarily symmetric about 
TABLE 1: Results for manoeuvres respective with horizontal zigzag sea trials as reported by Issac et al. [13].

\begin{tabular}{lccccccccccc}
\hline Test no. & $U(\mathrm{~m} / \mathrm{s})$ & $\delta_{0}(\mathrm{deg})$ & $\psi_{0}(\mathrm{deg})$ & $T_{p}(\mathrm{~s})$ & $A_{z}(\mathrm{~m})$ & $L_{z}(\mathrm{~m})$ & $t_{a}(\mathrm{~s})$ & $\alpha_{s}(\mathrm{deg})$ & $d_{s}(\mathrm{~m})$ & $r_{0}(\mathrm{deg} / \mathrm{s})$ & $v_{0}(\mathrm{~m} / \mathrm{s})$ \\
\hline 1 & 1.5 & 1.5 & 22.5 & 112.8 & 9.5 & 158.7 & 26.8 & 2.2 & 13.8 & 1 & 0.05 \\
2 & 2 & 1.5 & 22.5 & 86.2 & 9.9 & 161.5 & 20.2 & 2.6 & 14.4 & 1.3 & 0.07 \\
3 & 1.5 & 2.6 & 45 & 137 & 20.7 & 161.7 & 30.9 & 5.1 & 29.8 & 1.6 & 0.08 \\
4 & 2 & 2.6 & 45 & 105.4 & 21.7 & 164.7 & 23.5 & 6.3 & 31.9 & 2.2 & 0.11 \\
\hline
\end{tabular}

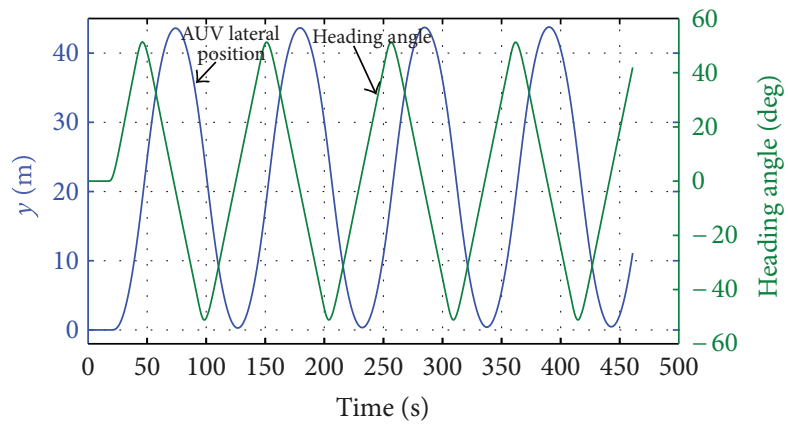

FIGURE 3: AUV lateral position and heading during horizontal zigzag; simulation at $U=2 \mathrm{~m} / \mathrm{s}, \delta=2.6 \mathrm{deg}$, and $\psi=45 \mathrm{deg}$ (test no. 4).

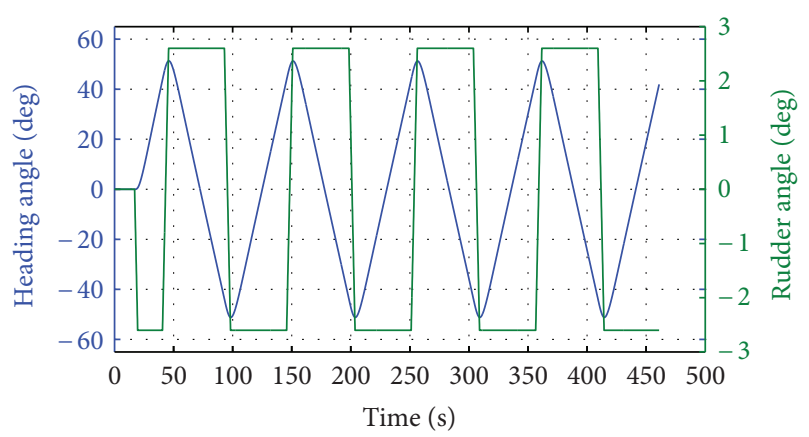

FIGURE 4: AUV heading and rudder deflection during horizontal zigzag; simulation at $U=2 \mathrm{~m} / \mathrm{s}, \delta=2.6 \mathrm{deg}$, and $\psi=45 \mathrm{deg}$ (test no. 4).

zero, will alternate between positive and negative amplitudes. Also note that the heading angle is zero when the lateral position of the AUV is an extreme (Figure 3). Finally, it is worthy to mention that the rudder deflection angle shown in Figure 4 is measured relative to the AUV hull; the apparent angle of attack relative to flow is a different curve [9].

\subsection{Deriving the Nomoto Indices Using the Simulation Model.} The Nomoto indices were extracted from the simulation model through the following procedure:

(i) According to (2), when the yaw angular acceleration of the AUV equals zero, the $K$ Nomoto index can be calculated as follows.

$$
K=\frac{r}{\delta},
$$

where $r$ is in deg/s and $\delta$ is in degrees; therefore, $K$ has the dimension of $\sec ^{-1}$. Note that the yaw angular acceleration

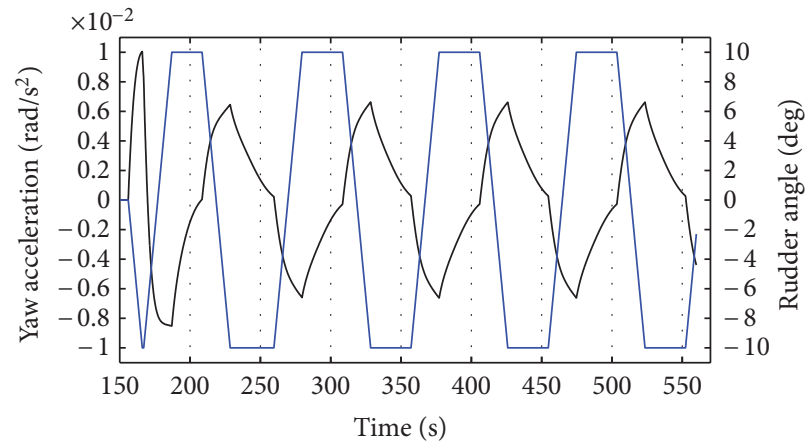

FIgURE 5: Rudder deflection and yaw angular acceleration during a 10-20 zigzag simulation run.

is constant at zero during the first stage of the manoeuvre (explained in Section 3.4); however, that portion of the manoeuvre is of no interest. After the zigzag starts, as the rudder deflection angle increases, the yaw angular acceleration increases and then decreases as the rudder deflection is kept constant and becomes negative as the rudder angle is reversed. Figure 5 shows the rudder deflection angle along with the yaw angular acceleration of the AUV on the same plot during the 10-20 zigzag run. The rudder angle is divided by 10000; the yaw angular acceleration is in $\mathrm{rad} / \mathrm{sec}^{2}$.

The first time during the zigzag that the yaw angular acceleration decreases to zero, (7); is used to calculate the $K$ Nomoto index. Since the simulation model is a discrete stepby-step calculation, one cannot expect an exact zero value for the acceleration at any particular time-step, instead if the acceleration reduces to one percent of its maximum value, the $K$ Nomoto index for that manoeuvre is recorded.

(ii) After the zigzag run is performed and the vectors of yaw rate of turn and acceleration are recorded over the zigzag duration, if the $K$ Nomoto index, that was calculated in stage 1 previously, is substituted into (2), then the $T$ Nomoto index is derived as a vector during the zigzag using the following relation:

$$
T(t)=\frac{[K \delta(t)-r(t)]}{\dot{r}(t)} .
$$

Note that, except for $K$, all the variables in (8) are functions of time during the zigzag; $T$ has dimension of time. Figure 6 shows the $T$ Nomoto index versus time during the 10-20 zigzag run. The diagram has two jumps on every cycle where the yaw angular acceleration decreases close to zero.

(iii) The nondimensional Nomoto index $T^{\prime}$ is defined as the mean value of the vector that was calculated in (8) 


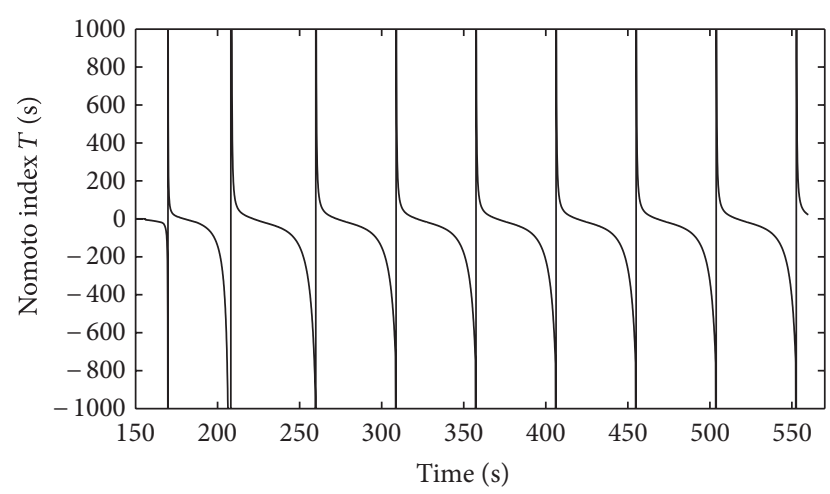

Figure 6: Nomoto index $T$ versus time calculated using (8), for approach speed $1.5 \mathrm{~m} / \mathrm{s}$ during a $10-20$ zigzag run.

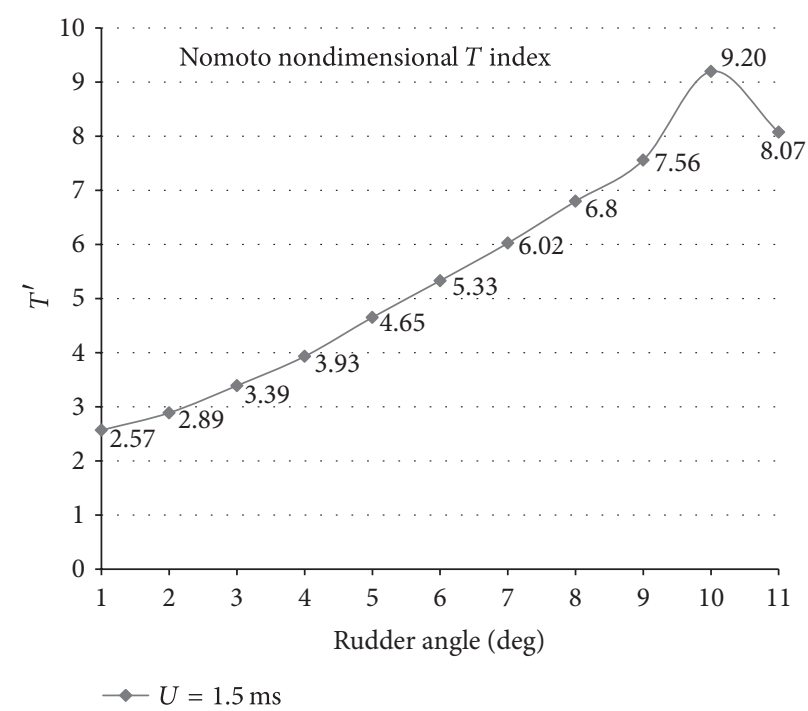

FIgURE 7: Nomoto nondimensional index $T^{\prime}$ versus rudder deflection angle for approach speed $1.5 \mathrm{~m} / \mathrm{s}$ and command heading 20 degrees.

and plotted in Figure 6, over the first two seconds in the "constant rudder deflection angle" period of the zigzag, times the approach speed divided by the AUV length. As has been explained before, during the zigzag, as the rudder starts to deflect, the yaw angular acceleration initiates from zero; therefore, the vector of Nomoto index that was defined in (8) has a jump during that period. Also, as the zigzag execute reaches its end, the yaw angular acceleration decreases to zero, and thus the vector of the $T$ Nomoto index that was defined in (8) has a jump during that period too. The most stable period of time during the zigzag cycle to calculate the $T$ Nomoto index, as mentioned, is early during the interval in which the constant rudder deflection angle is applied.

According to simulation results, it seems that the $K^{\prime}$ Nomoto index does not depend on either the rudder commanded angle, the desired heading angle, or the approach speed and has the same value of about two for all zigzag runs. In fact, this is what should be expected: the Nomoto indices are constant values indicative of manoeuvring characteristics of the vehicle. Moreover, the $T^{\prime}$ Nomoto index, as calculated in this simulation model, seems not to depend on the specified heading angle; however, it does depend on the commanded rudder angle (Figure 7) and also depends on the approach speed. At four degrees rudder command angle, $T^{\prime}$ is about four for $1.5 \mathrm{~m} / \mathrm{s}$ and about five for $2 \mathrm{~m} / \mathrm{s}$.

\section{Solution of the Nomoto's First-Order Model for a Square-Wave Input for the Rudder Deflection Angle}

The rudder deflection angle during a zigzag manoeuvre may be modeled as a square wave of amplitude $\delta_{0}$, defined as follows:

$$
\delta(t)= \begin{cases}-\delta_{0}, & -L<t<0 \\ \delta_{0}, & 0<t<L\end{cases}
$$

where $L$ in the previous equation equals half the period of the zigzag, $L=T_{Z} / 2$.

The first-order Nomoto model $T \dot{r}+r=K \delta$ has the following general solution:

$$
r(t)=e^{-t / T}\left[\int_{0}^{t} \frac{K}{T} \delta(t) e^{t / T} d t+c\right],
$$

where $K$ and $T$ are Nomoto indices, and $c$ is a constant that depends on the initial conditions of the problem; here, assume that the initial rate of turn of the vehicle is zero, $r(0)=0$.

To evaluate the integral inside the brackets in (10), the Fourier series representation of $\delta(t)$ in (9) is written as follows:

$$
\delta(t)=\frac{4 \delta_{0}}{\pi}\left[\sin \frac{\pi}{L} t+\frac{1}{3} \sin \frac{3 \pi}{L} t+\frac{1}{5} \sin \frac{5 \pi}{L} t+\cdots\right] .
$$

Substituting the rudder deflection angle from (11) into (10), using part-by-part integration, the rate of turn of the vehicle during such zigzag manoeuvre is obtained as follows:

$$
\begin{aligned}
r(t)=\frac{K}{T} \times \frac{4 \delta_{0}}{\pi} & \\
& \times \sum_{n=0}^{\infty}\left\{\frac{L}{(2 n+1)^{2} \pi}\right. \\
& \times\left[\frac{L}{(2 n+1) \pi T} \sin \frac{(2 n+1) \pi}{L} t\right. \\
& \times\left(1+\left(\frac{\left.-\cos \frac{(2 n+1) \pi}{(2 n+1) \pi T} t\right)}{L}\right)^{2}\right\} \\
& +c \times e^{-t / T} .
\end{aligned}
$$




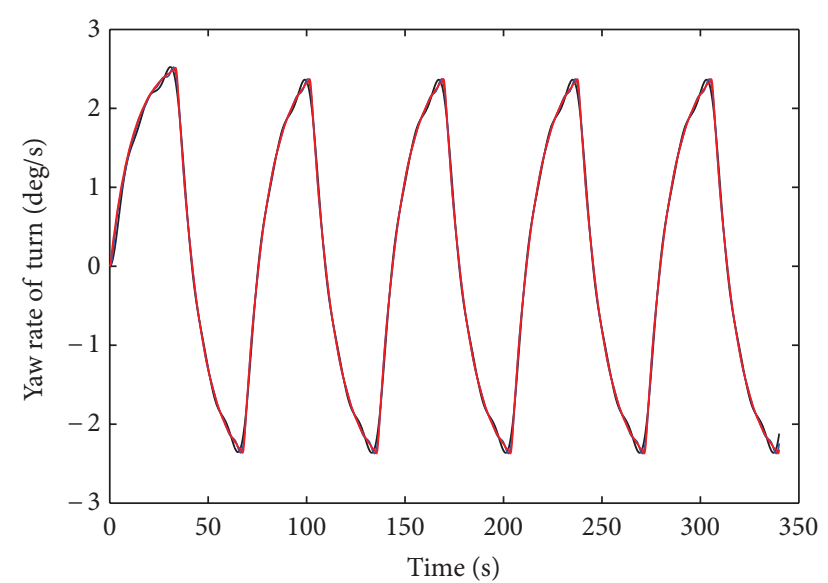

Equation (12) plotted for respectively,

$$
\begin{aligned}
N & =0 \text { to } 2 \\
N & =0 \text { to } 5 \\
N & =0 \text { to } 15
\end{aligned}
$$

FIGURE 8: The analytic solution using (12) for the yaw rate of turn for a $(4,20)$ zigzag at approach speed $1.5 \mathrm{~m} / \mathrm{s}$.

Then, the constant $c$ in (12) is calculated using $r(0)=0$, as follows:

$$
c=-\frac{K}{T} \times \frac{4 \delta_{0}}{\pi} \sum_{n=0}^{\infty}\left\{\frac{-L /(2 n+1)^{2} \pi}{\left[1+(L /(2 n+1) \pi T)^{2}\right]}\right\} .
$$

The inputs to (12) are the amplitude of rudder deflection angle and period of zigzag, as well as the Nomoto indices $K$ and $T$. The results of the simulation model are used for these inputs; for example, a $(4,20)$ zigzag at approach speed $1.5 \mathrm{~m} / \mathrm{s}$ results in nondimensional $K^{\prime}$ and $T^{\prime}$ values of about two and four, respectively, and a zigzag period of about 68 seconds. Using those inputs, (12) is solved by a recursive code which iterates the summation for arbitrary number of terms, $N$. Yaw rate of turn is then plotted in Figure 8 versus time. There are three different curves that respectively include two, five and 15 terms in the summation of the solution in (12). The period of the curve of turning rate of the vehicle equals the period of the input square wave, that is 68 seconds. If the peaks of the solution are zoomed in, as shown in Figure 9, the effect of number of terms in the summation is better observable. The fluctuations at the peaks of the turning rate curve, as seen in Figure 9, are known as the Gibbs phenomenon, the manner in which the Fourier series of a piecewise continuously differentiable periodic function behaves at a jump discontinuity [14].

The simulation result for turning rate for this manoeuvre is $2.4 \mathrm{deg} / \mathrm{s}$ amplitude. The analytic solution for this manoeuvre, (12) plotted in Figure 8, is showing closely the same result. The analytic values for the amplitude of rate of turn of the AUV for couple of manoeuvres were compared with the simulation model results. Although, the amplitudes of analytic and numeric solutions are in close agreement, there is phase difference between them, because the former uses a

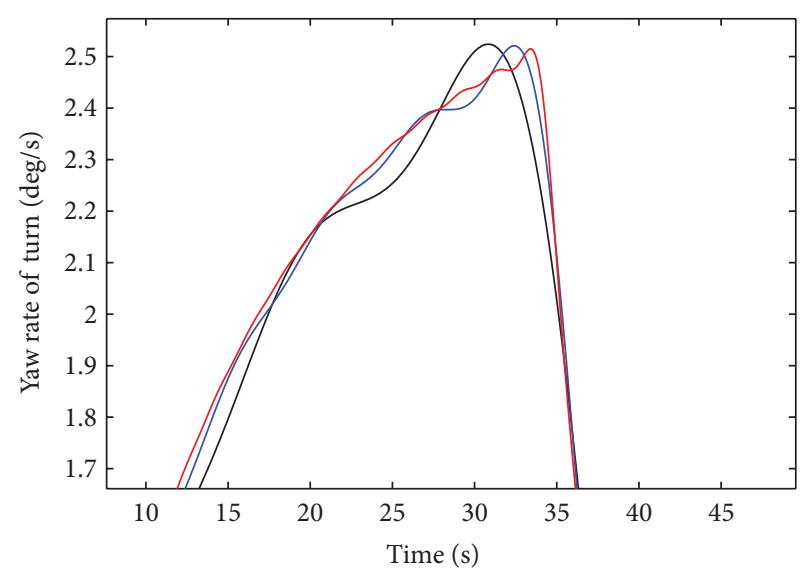

Equation (12) plotted for respectively,

$$
\begin{aligned}
N & =0 \text { to } 2 \\
-N & =0 \text { to } 5 \\
N & =0 \text { to } 15
\end{aligned}
$$

FIGURE 9: Figure 8 zoomed in showing the fluctuations at the peaks due to the number of terms in the summation in (12).

square-wave input and the latter uses a trapezoid-wave input as is instructed by ITTC test procedure.

Regarding the zigzag sea trials that were introduced in Section 4.2 and Table 1, using the simulation model, the value of nondimensional $K^{\prime}$ is the same about 1.96 and for $T^{\prime}$ respective values for test numbers one to four are 2.7, 3.2, 3.15 , and 3.9. Inserting these values along with the respective values of amplitude of rudder deflection angle and period of zigzag from Table 1 into (12) results in yaw rate of turns of 1 , $1.3,1.6$, and $2.2 \mathrm{deg} / \mathrm{s}$ which, respectively, exactly stick to the simulation values.

\section{Conclusions}

The concluding remarks are that zigzag manoeuvres of an underwater vehicle at a constant depth may be simulated by a numerical computer model by first modeling the hydrodynamics of the bare-hull, the control planes and the propeller and then solving the equations of motion of the vehicle as a time-step integration. The resulting simulation model should be verified using sea trials data which was done for the MUN Explorer AUV in this paper. On the other hand, Nomoto's first-order model for the rate of turn of the vehicle during horizontal zigzag manoeuvres in response to a square-wave input for the rudder deflection angle could be solved for Nomoto indices $K$ and $T$ using a Fourier series representation of the rudder deflection angle. In this paper, since the curve of response of the AUV (including its rate of turn) during zigzag manoeuvres was solved numerically, as explained previously, approximate values for the Nomoto indices were extracted during each run. Then, the indices were substituted in the analytic solution for the rate of turn, as explained previously, and the results were compared with the results of simulation model. Although the two approaches are completely independent (only the indices that were extracted from simulation model were substituted in 
the analytic solution), the results are fairly the same. Of course, in practice, the trapezoidal-wave approach follows the standard test procedure as recommended by the ITTC. Results of this research are the first step to understand details of zigzag manoeuvres of an AUV. The next step should be taken if a more accurate model of the AUV hydrodynamics is developed. Then, the manoeuvres may be studied in threedimensional underwater space.

\section{Acknowledgments}

The authors would like to thank the Memorial University of Newfoundland, the Institute for Ocean Technology, National Research Council Canada (NRC-IOT), and the National Science and Engineering Research Council (NSERC), as well as the Islamic Azad University (IAU) for their support of this research.

\section{References}

[1] P. Mandel, "ship maneuvering and control," in Principles of Naval Architecture, J. P. Comstock, Ed., chapter 8, Society of Naval Architects and Marine Engineers, 1967.

[2] "Full scale manoeuvring trials procedure," in The Proceedings of the 23rd International Towing Tank Conference (ITTC '02), ITTC Quality Manual, 4.9-03/04-01, 2002.

[3] D. Perrault, N. Bose, S. O’Young, and C. D. Williams, "Sensitivity of AUV response to variations in hydrodynamic parameters," Ocean Engineering, vol. 30, no. 6, pp. 779-811, 2003.

[4] L. Moreira and C. G. Soares, "Dynamic model of manoeuvrability using recursive neural networks," Ocean Engineering, vol. 30, no. 13, pp. 1669-1697, 2003.

[5] S. Sutulo and C. G. Soares, "Numerical study of some properties of generic mathematical models of directionally unstable ships," Ocean Engineering, vol. 32, no. 3-4, pp. 485-497, 2005.

[6] F. L. Pérez and J. A. Clemente, "The influence of some ship parameters on manoeuvrability studied at the design stage," Ocean Engineering, vol. 34, no. 3-4, pp. 518-525, 2007.

[7] J. M. J. Journée, "A simple method for determining the manoeuvring indices K and T from zigzag trial data," Report 267, Delft University of Technology, Ship Hydromechanics Laboratory, 1970.

[8] M. T. Issac, S. Adams, M. He et al., "Manoeuvring experiments using the MUN Explorer AUV," in Proceedings of the Symposium on Underwater Technology and Workshop on Scientific Use of Submarine Cables and Related Technologies, pp. 256-262, Tokyo, Japan, April 2007.

[9] F. Azarsina and C. D. Williams, "Manoeuvring simulation of the MUN Explorer AUV based on the empirical hydrodynamics of axi-symmetric bare hulls," Applied Ocean Research, vol. 32, no. 4, pp. 443-453, 2010.

[10] K. Nomoto, "Analysis of Kempf's standard maneuver test and proposed steering quality indices," in Proceedings of the 1st Symposium in Ship Maneuverability, DTRC Report 1461, 1960.

[11] K. Nomoto, Response Analysis of Maneuverability and Its Application To Ship Design, Researches on the Maneuverability of Ships in Japan, Society of Naval Architects of Japan, Tokyo, Japan, 1966.

[12] Explorer webpage, http://www.mun.ca/research/ocp/creait/ merlin/auv.php.
[13] M. T. Issac, S. Adams, N. Bose, C. D. Williams, R. Bachmayer, and T. Crees, "Analysis of horizontal zigzag manoeuvring trials from the MUN Explorer AUV," in Proceedings of the OCEANS, Quebec City, Canada, September 2008.

[14] E. Kreyszig, Advanced Engineering Mathematics, John Wiley \& Sons, New York, NY, USA, 2006. 

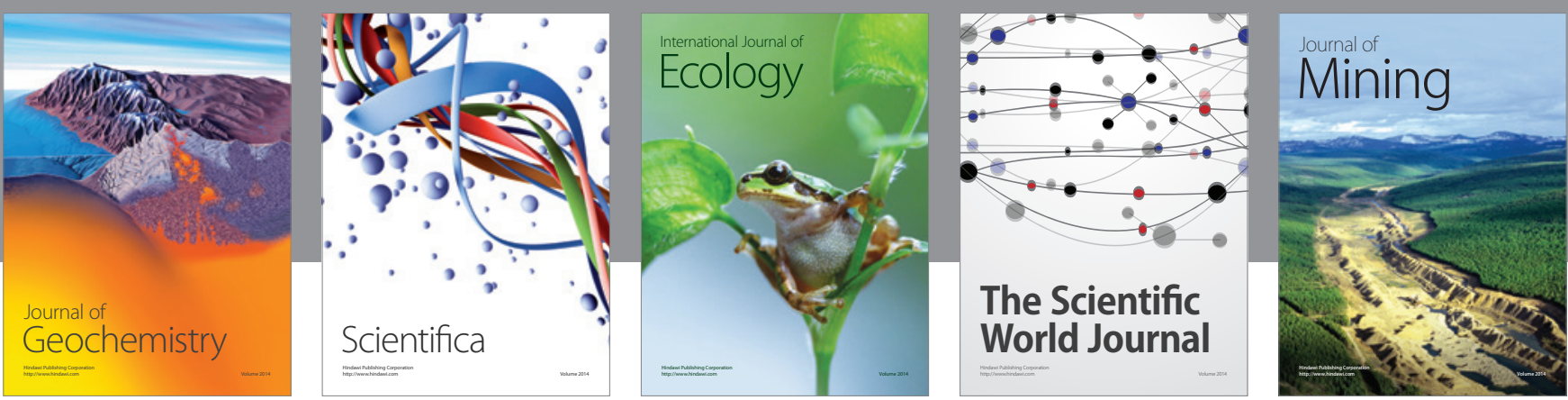

The Scientific World Journal
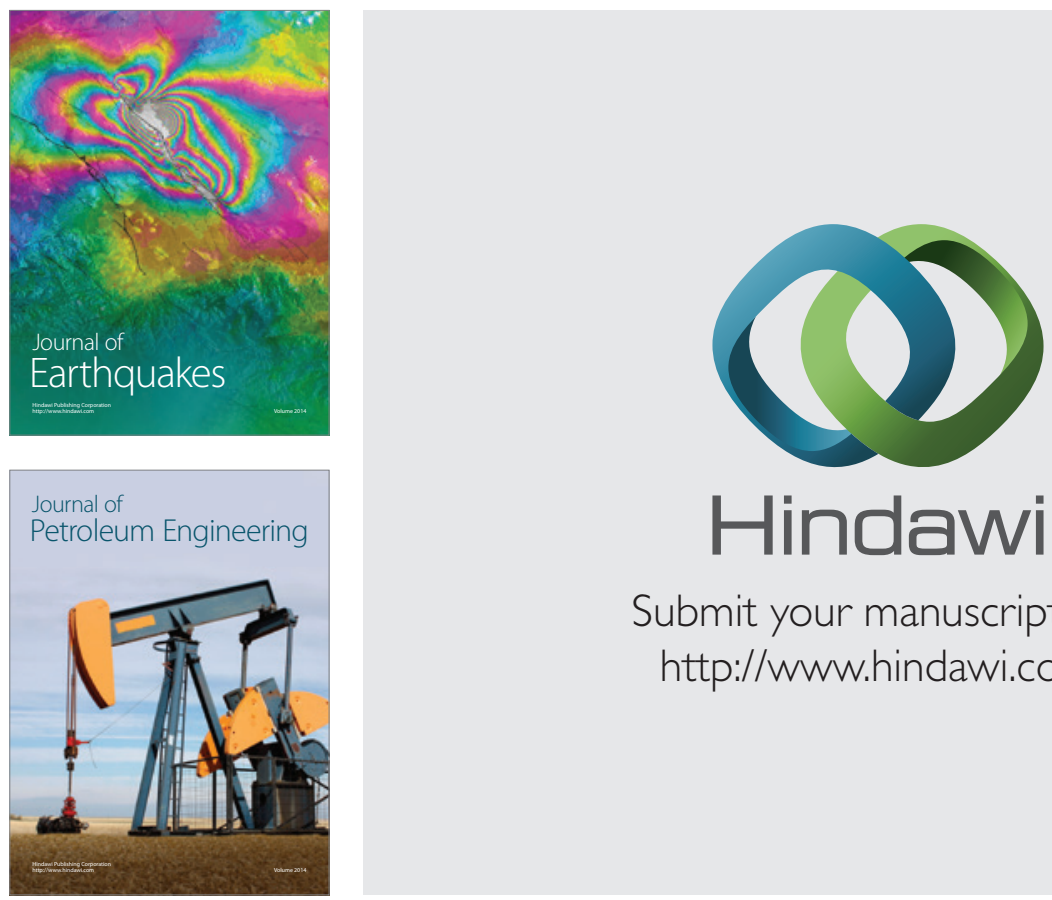

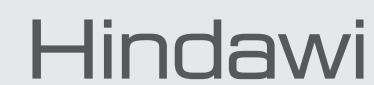

Submit your manuscripts at

http://www.hindawi.com
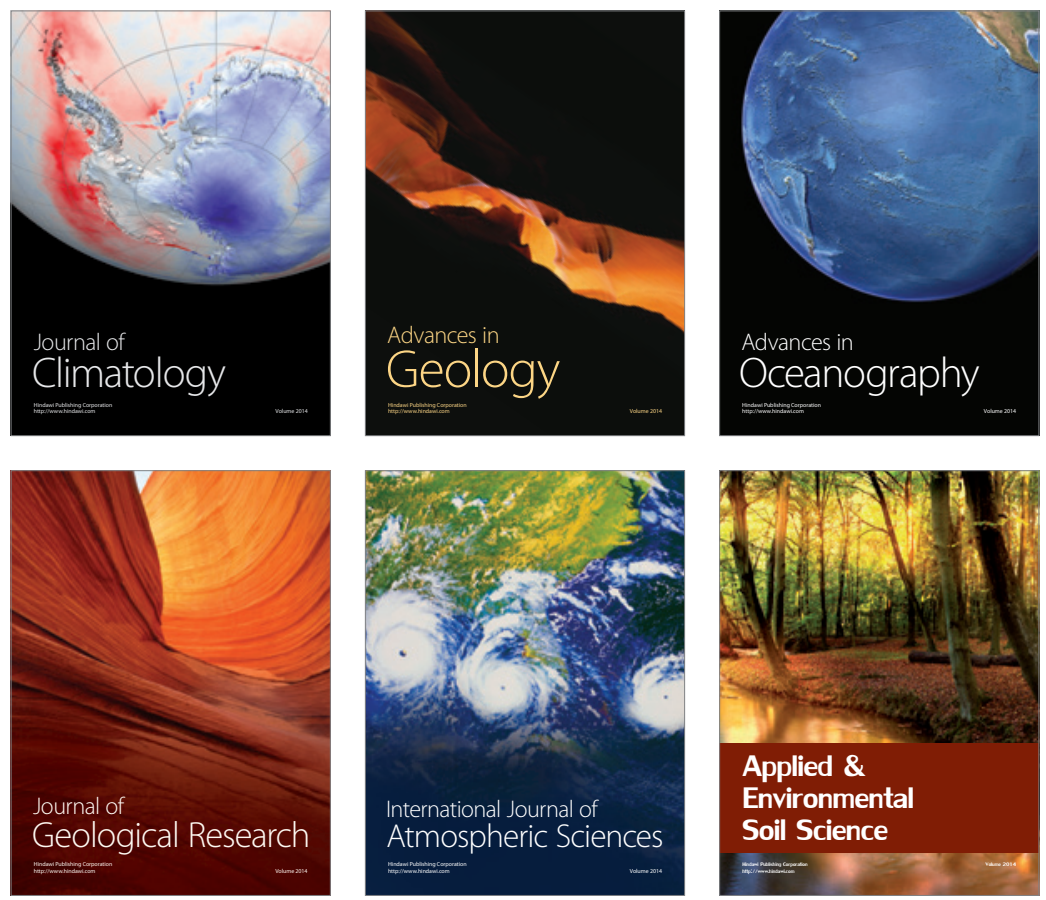
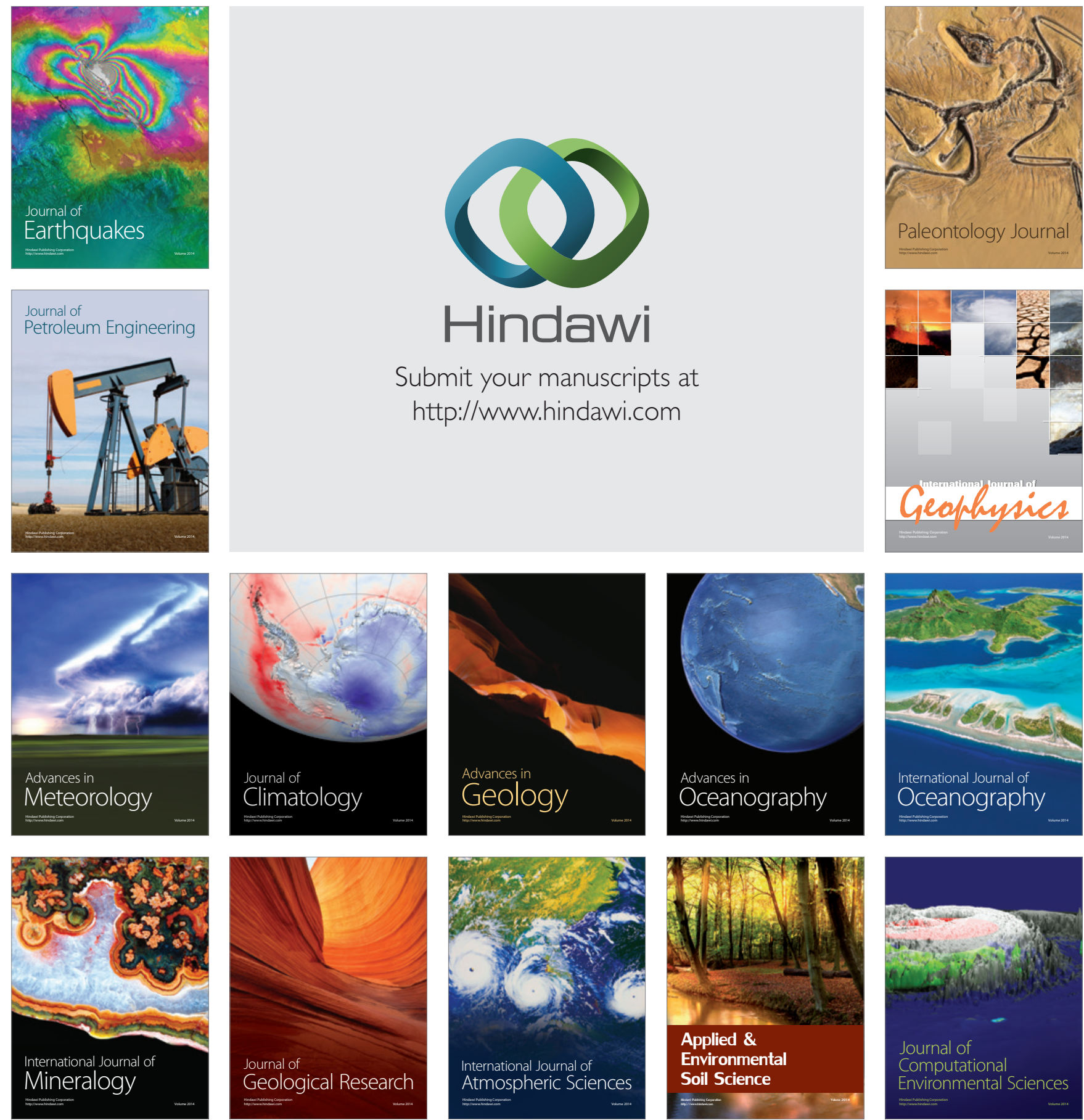